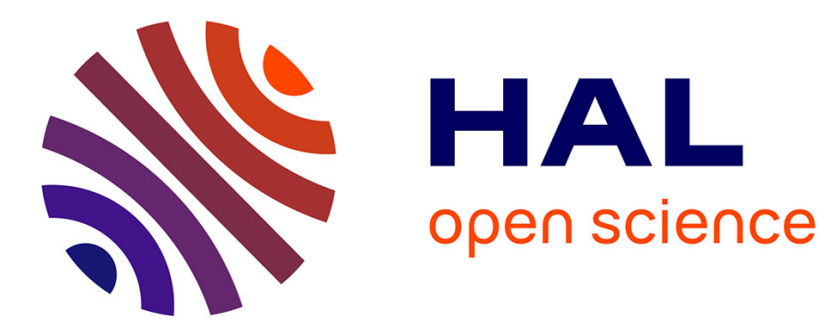

\title{
Upscaling of Anomalous Pore-Scale Dispersion
}

Alexandre Puyguiraud, Philippe Gouze, Marco Dentz

\section{To cite this version:}

Alexandre Puyguiraud, Philippe Gouze, Marco Dentz. Upscaling of Anomalous Pore-Scale Dispersion.

Transport in Porous Media, 2019, 128 (2), pp.837-855. 10.1007/s11242-019-01273-3 . hal-03092703

\section{HAL Id: hal-03092703 \\ https://hal.science/hal-03092703}

Submitted on 2 Jan 2021

HAL is a multi-disciplinary open access archive for the deposit and dissemination of scientific research documents, whether they are published or not. The documents may come from teaching and research institutions in France or abroad, or from public or private research centers.
L'archive ouverte pluridisciplinaire HAL, est destinée au dépôt et à la diffusion de documents scientifiques de niveau recherche, publiés ou non, émanant des établissements d'enseignement et de recherche français ou étrangers, des laboratoires publics ou privés. 


\title{
Upscaling of anomalous pore-scale dispersion ${ }^{\star}$
}

\author{
Alexandre Puyguiraud, Philippe Gouze and \\ Marco Dentz
}

Received: date / Accepted: date

\begin{abstract}
We study the upscaling of advective pore-scale dispersion in terms of the Eulerian velocity distribution and advective tortuosity, both flow attributes, and of the average pore length, a medium attribute. The stochastic particle motion is modeled as a time-domain random walk, in which particles move along streamlines in equidistant spatial steps with random velocities and thus random transition times. Particle velocities describe stationary spatial Markov processes, which evolve along streamlines on the mean pore length. The streamwise motion is projected onto the mean flow direction using tortuosity. This upscaled stochastic particle model predicts accurately the (non-Fickian) transport dynamics obtained from direct numerical simulations of particle transport in a three-dimensional digitized Berea sandstone sample. It captures all aspects of transport and sheds light on the dependence of the upscaled transport behavior on the flow heterogeneity and the initial particle distribution, which are critical for the accurate modeling of dispersion from the pre-asymptotic to asymptotic regimes.
\end{abstract}

* Puyguiraud, A., P. Gouze, and M. Dentz, Upscaling of anomalous pore-scale dispersion, Transp. Porous Media, https://doi.org/10.1007/s11242-019-01273-3, 2019.

A. Puyguiraud

Spanish National Research Council (IDAEA-CSIC), 08034 Barcelona, Spain

Géosciences Montpellier, CNRS-Université de Montpellier, Montpellier, France

E-mail: alexandre.puyguiraud@cid.csic.es

P. Gouze

Géosciences Montpellier, CNRS-Université de Montpellier, Montpellier, France

E-mail: philippe.gouze@univ-montp2.fr

M. Dentz

Spanish National Research Council (IDAEA-CSIC), 08034 Barcelona, Spain

E-mail: marco.dentz@csic.es 


\section{Introduction}

Upscaling hydrodynamic transport is a critical step for modeling solute dispersion in porous media. Since the pioneering works of de Josselin de Jong (1958) and Saffman (1959), different approaches have been used for deriving dispersion coefficients and advection-dispersion models for asymptotic spreading and mixing in heterogeneous media (Bear, 1972; Brenner and Edwards, 1993; Salles et al., 1993; Whitaker, 1999). However, the asymptotic regime is often not reached for space and time scales relevant at the laboratory scale, for environmental or industrial applications (Levy and Berkowitz, 2003; Le Borgne and Gouze, 2008; Moroni et al., 2007), which makes it important to account for pre-asymptotic transport, which can in general not be characterized by constant hydrodynamic dispersion, and thus may be termed non-Fickian or anomalous. Non-Fickian pre-asymptotic dispersion is caused by incomplete mixing on the support scale and thus incomplete sampling of the velocity heterogeneity due to spatial heterogeneity, which characterizes natural systems (Dentz et al., 2000, 2004; Berkowitz et al., 2006; Nicolaides et al., 2010; Wood, 2009; Le Borgne et al., 2011; Dentz et al., 2011). The conditions under which the behavior can asymptotically be described by hydrodynamic dispersion and the transition to such a regime was discussed in Salles et al. (1993), Dentz et al. (2004), and Bijeljic and Blunt (2006). For systems characterized by large or infinite Péclet numbers, non-Fickian behavior may be related to a broad distribution of velocity values. Recent pore-scale transport studies (De Anna et al., 2013; Kang et al., 2014; Holzner et al., 2015; Morales et al., 2017; Carrel et al., 2018) showed that observed intermittency of temporal velocity series along individual streamlines are closely related to the occurrence of anomalous dispersion.

The quantification of pre-asymptotic dispersion and its causes in the medium and flow properties is a critical issue for upscaling hydrodynamic transport from the pore to the Darcy scale. Pre-asymptotic (non-Fickian) dispersion on the pore and Darcy scales have been modeled by a variety of non-local approaches (Neuman and Tartakovsky, 2009), such as the multirate mass transfer (MRMT) approach (Haggerty and Gorelick, 1995; Carrera et al., 1998), volume averaging and two-equation formulations for transport (Cherblanc et al., 2007; Davit et al., 2010; Porta et al., 2013), the continuous time and time-domain random walk approaches (Berkowitz and Scher, 1995; Dentz and Berkowitz, 2003; Berkowitz et al., 2006; Bijeljic and Blunt, 2006; Wright et al., 2019; Sund et al., 2015, 2017; Sherman et al., 2019), see also the recent review by Noetinger et al. (2016). A critical step for implementing these non-local models concerns the relation between the velocity statistics that are controlled by the pore-scale structure, and the macroscopic transport process. Porta et al. (2015) derived a mobile-immobile model to upscale pore-scale transport accounting for information on the pore space and the pore-scale velocity distributions. Meyer and Bijeljic (2016) used a Langevin approach to account for the impact of pore-scale velocity heterogeneity on solute dispersion. Due to their central role for transport upscaling from the pore to the Darcy scale, pore-scale particle velocities and their relation to the flow velocity and porous medium structure have been the subject of recent research (De Anna et al., 2013; Siena et al., 2014; Holzner et al., 2015; Morales 
et al., 2017; Jin et al., 2016; Matyka et al., 2016; De Anna et al., 2017; Dentz et al., 2018).

A strategy to systematically upscale (advective) transport from the pore to the Darcy scale consists in identifying the stochastic dynamics of particle velocities, formalizing the link between Lagrangian and Eulerian statistics, and relating the flow statistics to statistical pore-scale properties. (Morales et al., 2017) and Puyguiraud et al. (2019) linked observed intermittent patterns in the temporal velocity series to the spatial persistence of pore-scale velocities. These authors showed that velocity series sampled equidistantly along streamlines do not exhibit such intermittent patterns and can be represented by a spatial Markov process. Puyguiraud et al. (2019) showed for the Berea sandstone sample under consideration in this paper that the spatial velocity series can be represented by an ergodic and stationary Markov process at the sample scale. This observation implies that upscaled transport can be understood and modeled in terms of time-domain or continous time random walks.

In this paper we use the representation of equidistant particle velocities as a stationary Markov process to upscale particle motion and solute transport in the framework of time-domain random walks in terms of the pore-scale velocity distribution and characteristic length scale. We employ two velocity Markov models of different complexity. The first is based on a Bernoulli process for the prediction of the velocity series, the second on an Ornstein-Uhlenbeck velocity process for the normal scores of velocity (Morales et al., 2017; Puyguiraud et al., 2019). The resulting timedomain random walk models are used to predict breakthrough curves, displacement mean and variance as well as the full spatial particle distributions or propagators from full three-dimensional flow and particle tracking simulations for a Berea sandstone sample.

The paper is organized as follows. The methodology we use is detailed in Section 2. We specify the flow equation and the transport equation that are solved, and summarize the details about the image acquisition, the flow simulation and the particle tracking simulations. Then, we present the stochastic particle model and describe the parameterization of the velocity process. In Section 3, we compare the transport data of the three-dimensional direct particle tracking simulations to the predictions of the upscaled models for uniform and flux weighted injection modes. The conclusions are presented in Section 4.

\section{Methodology}

In this section, we first present the basic equations for the three-dimensional direct numerical simulation (DNS) of flow and particle motion (particle tracking simulations) at pore scale. Then, we detail the upscaling methodology in the framework of a stochastic model and finally, we provide a summary of the numerical methodology.

\subsection{Flow and Particle Motion}

The Navier-Stokes momentum balance equation is classically used to model porescale flow $\mathbf{v}(\mathbf{x})$ of an incompressible fluid. At low values of the Reynolds number, the 
inertial forces are negligible in comparison to the viscous forces and the momentum equation reduce to, Leal (2007):

$$
\nabla^{2} \mathbf{v}(\mathbf{x})=\frac{1}{v} \nabla p(\mathbf{x})
$$

where $p(\mathbf{x})$ and $v$ denote the pressure and the kinematic viscosity of the fluid, respectively. The associated mass conservation equation is $\nabla \cdot \mathbf{v}(\mathbf{x})=0$. The position vector is denoted by $\mathbf{x}=\left(x_{1}, x_{2}, x_{3}\right)^{\top}$. The problem is solved by fixing constant pressure at both the inlet and outlet boundaries of the sample and no-slip condition at the voidsolid interfaces and at the other physical boundaries of the sample. Details concerning the computations and sample characteristics are given in section 2.3. The magnitude of the Eulerian velocity $\mathbf{v}(\mathbf{x})$ in the following is denoted by $v_{e}(\mathbf{x})=\|\mathbf{v}(\mathbf{x})\|$. The probability density function (PDF) of $v_{e}(\mathbf{x})$ is denoted by $p_{e}(v)$. It can be obtained by spatial sampling over a sampling volume that is representative of flow variability.

We consider purely advective transport. Thus, the trajectory of a particle originally located at $\mathbf{x}(t=0, \mathbf{a})=\mathbf{a}$ is described by

$$
\frac{d \mathbf{x}(t, \mathbf{a})}{d t}=\mathbf{v}[\mathbf{x}(t, \mathbf{a})]
$$

where $\mathbf{v}[\mathbf{x}(t, \mathbf{a})]$ is the Lagrangian velocity. Its magnitude is $v_{t}(t, \mathbf{a})=\|\mathbf{v}[\mathbf{x}(t, \mathbf{a})]\|$. The travel distance $s(t, \mathbf{a})$ along a particle trajectory until time $t$ and the travel time $t(s, \mathbf{a})$ up to a streamwise distance $t(s, \mathbf{a})$ are given by

$$
\frac{d s(t, \mathbf{a})}{d t}=v_{t}(t, \mathbf{a}), \quad \frac{d t(s, \mathbf{a})}{d s}=\frac{1}{v_{s}(s, \mathbf{a})},
$$

where we defined $v_{s}(s, \mathbf{a})=v_{t}[t(s), \mathbf{a}]$. We perform a variable change from time to streamwise distance, which renders time as $t(s, \mathbf{a})$ a dependent variable. The transform from $t \rightarrow s$ implies setting $d t=d s / v_{s}(s, \mathbf{a})$ in Eq. (2). This gives for the particle position as a function of distance $s$ the evolution equation

$$
\frac{d \hat{\mathbf{x}}(s, \mathbf{a})}{d s}=\omega(s, \mathbf{a}), \quad \omega(s, \mathbf{a})=\frac{\mathbf{v}[\hat{\mathbf{x}}(s, \mathbf{a})]}{v_{s}(s, \mathbf{a})},
$$

where $\omega(s, \mathbf{a})$ denotes the unit vector in the flow direction, $\mathbf{v}[\hat{\mathbf{x}}(s, \mathbf{a})]$ is denoted the s(pace)-Lagrangian velocity (Dentz et al., 2016; Puyguiraud et al., 2019) because it is the particle velocity at a given spatial distance $s$ along the particle trajectory, and its magnitude $\|\mathbf{v}[\mathbf{x}(s, \mathbf{a})]\|$ is equal to $v_{s}(s, \mathbf{a})$. We will refer in the following to $v_{s}(s)$ simply as particle velocity. Equation (3) describes the motion of a particle along a given streamline as a time-domain random walk (Painter and Cvetkovic, 2005; Noetinger et al., 2016) in that particles perform transitions over a fixed streamwise distance in variable time, which depends on the local velocity. Particle motion can be solved alternatively by integrating Eq. (2) in time or by integrating the system of equations (3) in streamwise distance. The numerical simulations performed in this paper use the former, the upscaling methodology presented in the next section uses the latter.

The distribution of initial particle positions is denoted by $\rho(\mathbf{a})$. We consider here two different initial particle distributions, uniform and flux-weighted, in order to 
probe the impact of the initial condition on average particle transport. The uniform and flux-weighted initial distribution read as

$$
\rho(\mathbf{a})=\frac{1}{V_{0}} \mathbb{I}\left(\mathbf{a} \in \Omega_{0}\right), \quad \rho(\mathbf{a})=\frac{v_{e}(\mathbf{a})}{\int_{\Omega_{0}} d \mathbf{x} v_{e}(\mathbf{x})} \mathbb{I}\left(\mathbf{a} \in \Omega_{0}\right),
$$

where $\Omega_{0}$ is the injection domain, $V_{0}$ its volume; $\mathbb{I}(\cdot)$ is the indicator function which is 1 if its argument is true and 0 otherwise.

In the following, we study the transport behavior in terms of breakthrough curves and spatial particle distributions and subsequently quantify dispersion by analyzing the first and second displacement moments. The breakthrough curve at a control plane located at position $x_{1}$ in the mean flow direction, is defined in terms of the first passage time

$$
\tau\left(x_{1}, \mathbf{a}\right)=\min \left[t \mid x_{1}(t, \mathbf{a}) \geq x_{1}\right] .
$$

where $x_{1}(t, \mathbf{a})$ denotes the position of particle a after time $t$ in mean flow direction. The breakthrough curve is equal to the PDF of the first passage times,

$$
f\left(t, x_{1}\right)=\int_{\Omega_{0}} d \mathbf{a} \rho(\mathbf{a}) \delta\left[t-\tau\left(x_{1}, \mathbf{a}\right)\right] .
$$

The breakthrough curves contain information on the residence times within the rock sample or the volume between the inlet and control plane, and the concentration in the effluent fluid. This information is useful for modeling reactive transport, for instance for applications to design aquifer decontamination or model laboratory dissolutionprecipitation experiments. Furthermore, we consider the spatial particle distribution, also called propagator, which is defined by

$$
g\left(x_{1}, t\right)=\int_{\Omega_{0}} d \mathbf{a} \rho(\mathbf{a}) \delta\left[x_{1}-x_{1}(t, \mathbf{a})\right] .
$$

This quantity gives information on the dispersion of a solute or particle cloud. Likewise this information can be used in the modeling of reactive transport and deployment of a reactant species, as well as for assessment of propagators in NMR imaging of flow and transport in porous media. The mean displacement and displacement variance are defined by

$$
\begin{aligned}
& m_{1}(t)=\int_{\Omega_{0}} d \mathbf{a} \rho(\mathbf{a}) x_{1}(t, \mathbf{a}), \\
& \sigma^{2}(t)=\int_{\Omega_{0}} d \mathbf{a} \rho(\mathbf{a})\left[x_{1}(t, \mathbf{a})-m_{1}(t)\right]^{2} .
\end{aligned}
$$

They measure the center of mass position and spatial variance of the particle distribution $g\left(x_{1}, t\right)$. The spatial variance is a measure for hydrodynamic dispersion. If its asymptotic evolution is linear, its growth rate is equal to the hydrodynamic dispersion coefficient. 


\subsection{Stochastic Model}

We formulate a stochastic model for particle motion in the mean flow direction $x_{1}$ of the coordinate system based on the stochastic representation of the s-Lagrangian velocity magnitude $v_{s}(s, \mathbf{a})$ as a stationary and ergodic Markov process $v_{s}(s)$ (Puyguiraud et al., 2019). The Markov process for $v_{s}(s)$ is characterized by the PDF $r\left(v, s-s^{\prime} \mid v^{\prime}\right)$ to make a transition from $v^{\prime}=v_{s}\left(s^{\prime}\right)$ at distance $s^{\prime}$ to $v=v_{s}(s)$ at $s>s^{\prime}$ and the steady state distribution $p_{s}(v)$. Both the velocity PDF $p(v, s)$ and the transition PDF $r\left(v, s \mid v^{\prime}\right)$ satisfy the Chapman-Kolmogorov equation (Risken, 1996)

$$
p(v, s)=\int_{0}^{\infty} r\left(v, s-s^{\prime} \mid v^{\prime}\right) p\left(v^{\prime}, s^{\prime}\right) d v^{\prime} .
$$

The transition probability converges to the steady-state distribution in the limit of $s \gg \ell_{c}$ with $\ell_{c}$ a characteristic velocity correlation scale,

$$
\lim _{s \rightarrow \infty} r\left(v, s \mid v^{\prime}\right)=p_{s}(v) .
$$

The characteristic correlation scale $\ell_{c} \approx 2.5 \ell_{p}$ where $\ell_{p}$ is the characteristic pore length. Eq. (11) implies that the distribution converges to the steady-state PDF $P(v)$ independently of the initial condition $p_{0}(v)$. The steady state distribution $p_{s}(v)$ is related to the Eulerian velocity PDF $p_{e}(v)$ through flux weighting (Dentz et al., 2016; Puyguiraud et al., 2019)

$$
p_{s}(v)=\frac{v p_{e}(v)}{\left\langle v_{e}\right\rangle}
$$

where $\left\langle v_{e}\right\rangle$ is the mean Eulerian velocity.

In this framework, the irregular particle motion described by (3) is represented by the stochastic evolution equations

$$
\frac{d \hat{x}_{1}(s)}{d s}=\chi^{-1}, \quad \frac{d t(s)}{d s}=\frac{1}{v_{s}(s)},
$$

where $\hat{x}_{1}(s)$ indicates the position of the particle in the mean flow direction (denoted by the subscript 1 similarly to Section 2.1). Note that the displacement rate $\omega_{1}(s)$ in 1 -direction in general fluctuates with $s$. We represent it here by its average $\left\langle\omega_{1}(s)\right\rangle=$ $\chi^{-1}$, where $\chi$ is the advective tortuosity given by (Koponen et al., 1996; Ghanbarian et al., 2013)

$$
\chi=\frac{\left\langle v_{e}(\mathbf{x})\right\rangle}{\left\langle v_{1}(\mathbf{x})\right\rangle} .
$$

The advective tortuosity compares the distance $s$ along the streamline with the average linear distance in the mean flow direction $\left\langle x_{1}(s)\right\rangle$, see Appendix A for details. It is an indicator fort the complexity of the pore space and gives information on advective excursions transverse to the mean flow direction.

The stochastic model (13) belongs to the continuous time random walk or timedomain random walk class of models because the time increment varies between 
random walk steps (Noetinger et al., 2016). Different initial particle distributions $\rho(\mathbf{a})$ are in this framework quantified in terms of the corresponding initial velocity distribution $p_{0}(v)$, this means in terms of the PDF of velocities $v_{e}(\mathbf{x})$ in the injection domain $\Omega_{0}$,

$$
p_{0}(v)=\frac{1}{V_{0}} \int_{\Omega_{0}} d \mathbf{a} \rho(\mathbf{a}) \delta\left[v-v_{e}(\mathbf{a})\right]
$$

The breakthrough curve $f\left(t, x_{1}\right)$ is in this framework given by

$$
f\left(t, x_{1}\right)=\left\langle\delta\left[t-t\left(x_{1} \chi\right)\right]\right\rangle,
$$

where the angular brackets denote the ensemble average over all particles and $t\left(x_{1} \chi\right)$ denotes the travel time over the streamlines distance $s=x_{1} \chi$. The mean displacement and its variance are

$$
\begin{aligned}
m_{1}(t) & =\left\langle\hat{x}_{1}[s(t)]\right\rangle, \\
\sigma^{2}(t) & =\left\langle\left[\hat{x}_{1}[s(t)]-m_{1}(t)\right]^{2}\right\rangle,
\end{aligned}
$$

where $s(t)=\max [s \mid t(s) \leq t]$. The spatial particle distribution is accordingly given by

$$
g\left(x_{1}, t\right)=\left\langle\delta\left(x_{1}-\hat{x}_{1}[s(t)]\right)\right\rangle .
$$

In the following, we briefly review two Markov processes, which model the evolution of $p(v, s)$ from arbitrary initial conditions, a Bernoulli velocity process (Dentz et al., 2016) and an Ornstein-Uhlenbeck process (Morales et al., 2017) for the evolution of the normal scores of velocity.

\subsubsection{Bernoulli Process}

This Markov process for the prediction of the fluid particle velocities is modeled as a Bernoulli process where the velocity changes after a distance $\Delta s$ according to a Bernoulli trial. This means that the particle velocity $v_{s}(s)$ does not change with probability $p_{B}(\Delta s)=\exp \left(-\Delta s / \ell_{c}\right)$ and changes randomly with probability $1-p_{B}(\Delta s)$ to a velocity which is sampled from the steady state PDF $p_{s}(v)$. The transition probability $r\left(v, \Delta s \mid v^{\prime}\right)$ is then expressed by (Dentz et al., 2016)

$$
r\left(v, \Delta s \mid v^{\prime}\right)=\exp \left(-\Delta s / \ell_{c}\right) \delta\left(v-v^{\prime}\right)+\left[1-\exp \left(-\Delta s / \ell_{c}\right)\right] P(v) .
$$

The Bernoulli process reproduces qualitatively the evolution of the Lagrangian velocity statistics, but underestimates the convergence rate of $p(v, s)$ toward its steady state at low velocities (Puyguiraud et al., 2019). The Bernoulli process does not account for any velocity dependence of the decorrelation rate and is therefore not capable of capturing the faster decorrelation of low velocities. Nevertheless, we consider the Bernoulli process as a possible evolution model for the particle velocities due to its simplicity. In the following, we refer to this model as Bernoulli model. 


\subsubsection{Ornstein-Uhlenbeck Process}

This velocity Markov process considers the evolution of the normal scores

$$
w(s)=\Phi^{-1}\left(\Pi\left[v_{s}(s)\right]\right) \equiv F\left[v_{s}(s)\right],
$$

where $\Phi(w)$ is the cumulative Gaussian distribution and $\Pi(v)$ the cumulative steady state velocity distribution,

$$
\Phi(w)=\frac{1+\operatorname{erf}(w / \sqrt{2})}{2}, \quad \Pi(v)=\int_{0}^{v} p_{s}\left(v^{\prime}\right) d v^{\prime}
$$

where $p_{s}(v)$ is the steady state velocity distribution. The normal scores $w(s)$ follow the Ornstein-Uhlenbeck process (Gardiner, 2010; Morales et al., 2017)

$$
\frac{d w(s)}{d s}=-\ell_{c}^{-1} w(s)+\sqrt{2 \ell_{c}^{-1}} \xi(s),
$$

where $\xi(s)$ is a Gaussian white noise characterized by zero mean $\langle\xi(t)\rangle=0$ and covariance $\left\langle\xi(s) \xi\left(s^{\prime}\right)\right\rangle=\delta\left(s-s^{\prime}\right)$. The Ornstein-Uhlenbeck process is a mean reverting process. In the absence of the noise terms, $w(s)$ relaxes exponentially fast towards 0. In the presence of noise, there is a steady state for $s \gg \ell_{c}$, at which $w(s) \sim \xi(s)$. The transition PDF for the Ornstein-Uhlenbeck process is given by (Gardiner, 2010)

$$
r_{w}\left(w, s \mid w^{\prime}\right)=\frac{\exp \left(-\frac{\left[w-w^{\prime} \exp \left(-s / \ell_{c}\right)\right]^{2}}{2\left[1-\exp \left(-2 s / \ell_{c}\right)\right]}\right)}{\sqrt{2 \pi\left[1-\exp \left(-2 s / \ell_{c}\right)\right]}}
$$

The velocity values $v_{s}(s)$ are obtained from $w(s)$ at any distance $s$ through the Smirnov transform (Devroye, 1986)

$$
v(s)=\Pi^{-1}(\Phi[w(s)]) \equiv F^{-1}[w(s)] .
$$

The velocity transition PDF $r\left(v, s \mid v^{\prime}\right)$ is thus given by

$$
r\left(v, s \mid v^{\prime}\right)=r_{w}\left[F(v), F\left(v^{\prime}\right)\right] \frac{d F(v)}{d v} .
$$

In the following, we refer to this model as the OU model.

The process (22) is implemented numerically via an Euler scheme as,

$$
w_{n+1}=w_{n}-\ell_{c}^{-1} w_{n} \Delta s+\sqrt{2 \ell_{s}^{-1} \Delta s} \xi_{n},
$$

where $w_{n}=w(n \Delta s)$ and $\xi_{n}$ is a Gaussian random variable with 0 mean and unit variance. Accurate results are obtained using a discretization $\Delta s \leq \ell_{c} / 10$. 


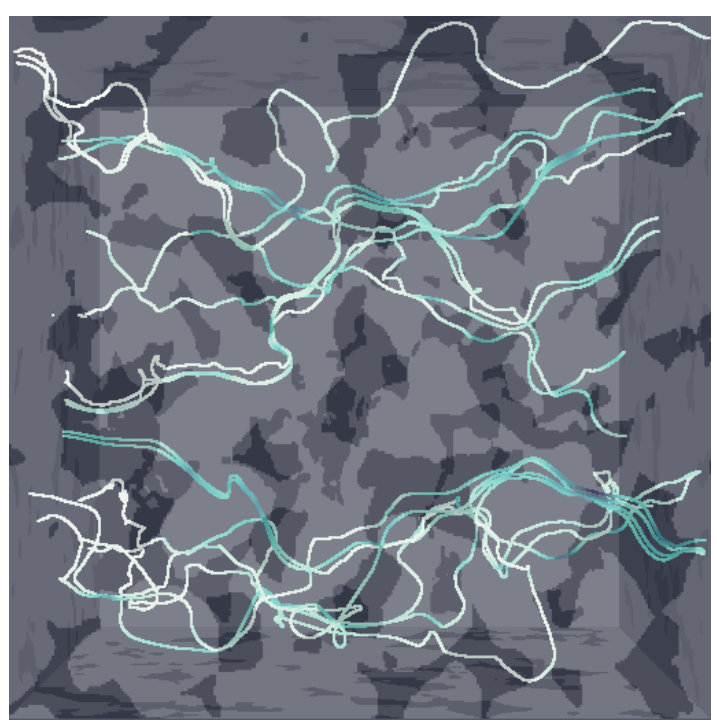

Fig. 1 Illustration of the subdomain of the Berea sandstone sample under consideration and sample streamlines.

\subsection{Rock Sample, Flow Field and Velocity Statistics}

Here we provide a brief summary of the rock sample, numerical methodology and velocity statistics. Details on the image acquisition and segmentation as well as the flow field computation can be found in Gjetvaj et al. (2015). Details regarding the particle tracking computation can be found in Puyguiraud et al. (2019).

We use a three-dimensional digitized image $\left(900^{3}\right.$ voxels) of a sample of a Berea sandstone (Upper Berea Sandstone unit, Ohio, USA). Berea sandstone is a sedimentary rock characterized by medium porosity and permeability values as well as medium pore-scale structural heterogeneity compared to common reservoir rocks. Because of these average properties, its simple composition (quasi pure silica) and its remarkable macroscopic homogeneity that allows easy comparisons, Berea sandstones are often used as a reservoir rock standard for experimental/ laboratory works. The image is reconstructed from X-ray microtomography (Paganin et al., 2002; Sanchez et al., 2012). The material density is recorded in a raw grey level image, which is is segmented (Smal et al., 2018) in order to obtain a binary image mapping the solid and the conne porosity. The porosity of the sample is 0.18 . The voxel length is $10^{-6}$ $\mathrm{m}$. The average pore length is $1.5 \cdot 10^{-4} \mathrm{~m}$.

The steady-state Navier-Stokes equations are solved using the SIMPLE method implemented in OpenFOAM (simpleFoam) (Weller et al., 1998) in order to obtain the velocity components at the center of the voxel surface for the full domain. The mean flow velocity is aligned with the 1-direction of the coordinate system and given by $\left\langle v_{1}\right\rangle=4.9 \cdot 10^{-4} \mathrm{~m} / \mathrm{s}$. The characteristic time scale is given by $\tau_{c}=\ell_{p} /\left\langle v_{1}\right\rangle=3 \cdot 10^{-1}$ $\mathrm{s}$. The streamlines starting at any location in $\Omega_{0}$ are built from the interpolated velocity using quadratic interpolation at the voxel in contact with the solid and linear 


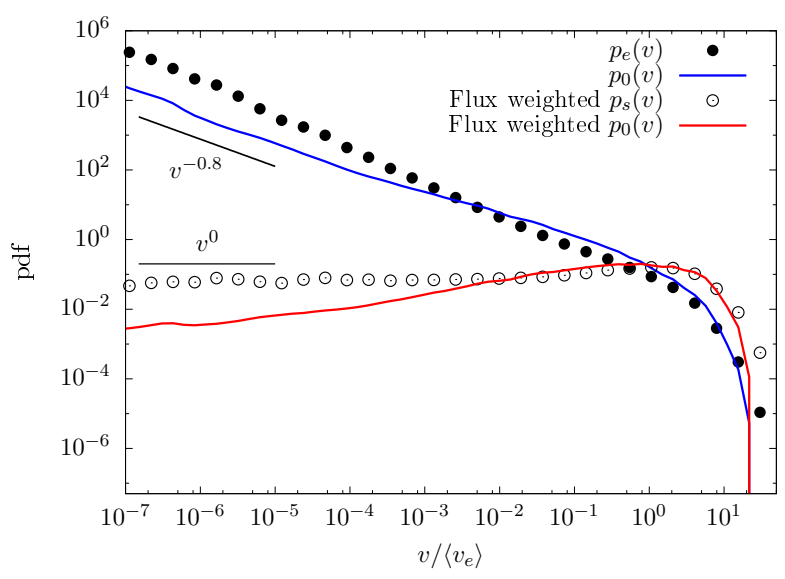

Fig. 2 Probability distribution function of the s-Lagrangian velocity, $p_{s}(v)$ (black empty circles), the initial velocities for uniform (blue) and flux-weighted (red) injections, $p_{0}(v)$, and the Eulerian velocity magnitude $p_{e}(v)$ (black full circles).

interpolation elsewhere (Pollock, 1988; Mostaghimi et al., 2012). The injection domain $\Omega_{0}$ is a box of an extension of 50 voxels in mean flow direction and 900 voxels in the directions perpendicular. Figure 1 illustrates a subdomain of the segmented Berea sandstone image and some example trajectories through the pore-space.

The stochastic models for particle motion described in the previous section require the knowledge of the velocity correlation length $\ell_{c}$, the steady state velocity $\mathrm{PDF} p_{s}(v)$ and the initial velocity PDF $p_{0}(v)$. Puyguiraud et al. (2019) performed a full statistical analysis of the velocity statistics of the rock sample under consideration. There, the velocity correlation length $\ell_{c}$ is found to be 2.5 times the characteristic pore length $\ell_{p}$. The mean Eulerian velocity magnitude is $\left\langle v_{e}\right\rangle=8.05 \cdot 10^{-4} \mathrm{~m} / \mathrm{s}$, which give the advective tortuosity $\chi=\left\langle v_{e}\right\rangle /\left\langle v_{1}\right\rangle=1.64$. The mean s-Lagrangian velocity magnitude is $\left\langle v_{s}\right\rangle=3.4 \cdot 10^{-3}$. The steady state velocity distribution $p_{s}(v)$, the Eulerian velocity distribution $p_{e}(v)$ and the initial velocity distributions for uniform and flux-weighted injections are shown in Figure 2. All velocity distributions show a strong tailing toward low velocities. For the flux-weighted injection, the initial velocity PDF $p_{0}(v)$ is close to the steady state PDF $p_{s}(v)$, while for the uniform injection, $p_{0}(v)$ is close to the Eulerian velocity PDF $p_{e}(v)$ (Puyguiraud et al., 2019). The next section studies particle transport through the sample using direct numerical simulations of purely advective particle motion, and its upscaling in terms of the velocity correlation length $\ell_{c}$ and velocity $\operatorname{PDF} p_{s}(v)$ in the framework of the velocity Markov models discussed in the previous section.

\section{Results}

We study here the upscaling of the purely advective particle motion in the Berea sandstone sample discussed in the previous section. Hydrodynamic transport in Berea sandstones is known to be non-Fickian at the scale of centimeter sized samples (Gjet- 
vaj et al., 2015; Bijeljic et al., 2011). The direct numerical flow and particle tracking simulations represent the reference data. The large scale behavior is measured in terms of particle breakthrough curves at different control planes, the particle displacement variance or dispersion, and the spatial particle distribution or propagators. These behaviors are then compared to the ones predicted by the stochastic particle models presented in the previous section, which quantify the upscaled particle motion.

\subsection{Breakthrough Curves}

The breakthrough curve denotes the residence time distribution of the solute in the domain. It may be used to infer the likeliness of chemical reactions to occur, and to assess the retention or storage potential of the subdomain, for example. Under uniform and homogeneous flow conditions, the BTC at control plane has an inverse Gaussian shape and decays sharply at long times. Under heterogeneous flow conditions breakthrough curves are characterized by early and late particle arrivals. In the following, we consider BTCs for flux-weighted and uniform injection conditions and compare them to the predictions of the upscaled transport models.

\subsubsection{Flux-Weighted Injection}

In this section we use a flux-weighted injection at the inlet for the computation of the breakthrough curves. The velocity PDF $p_{0}(v)$ at the inlet is close to the stationary PDF $p_{s}(v)$, see Figure 2. This implies that the particle velocities are approximately stationary. We compare the breakthrough curves of the direct simulation described in Section 2.3 to the two CTRW models described in Section 2.2. We perform the simulations using $10^{7}$ particles in the DNS case, while we used $10^{9}$ for the upscaled models. We compute arrival times at distances $x_{1}=6 \ell_{p}$ which corresponds to the end of the sample, $x_{1}=36 \ell_{p}$, and $x_{1}=200 \ell_{p}$. To compute the breakthrough curves at distances larger than the sample size, a particle exiting the sample at the outlet is reinjected at the inlet while conserving the velocity continuity (Puyguiraud et al., 2019).

Figure 3 displays the breakthrough curves from the DNS and the two stochastic models. We observe a strong anomalous behavior characterized by early peak arrivals and long tailing at late times. The late time tails display the power-law $t^{-2}$ at all distances. The exponent can be predicted from CTRW theory because it is directly linked to the behavior of the low velocity part of the steady Lagrangian velocity PDF, see Appendix B. The velocity distribution scales as $p_{s}(v) \propto v^{\beta-1}$ with $\beta=1$ for the small values of $v$. This implies that $f\left(t, x_{1}\right) \propto t^{-2}$.

The Bernoulli and OU models perform equally well. The early, intermediate, and late times are well captured even if at the closest control plane the two models do not reproduce the first arrivals perfectly. The two models give similar results because the injection velocity PDF is close to the steady-state PDF and therefore the models only need to be able to preserve this distribution over time, which they are both capable of doing (Puyguiraud et al., 2019). 


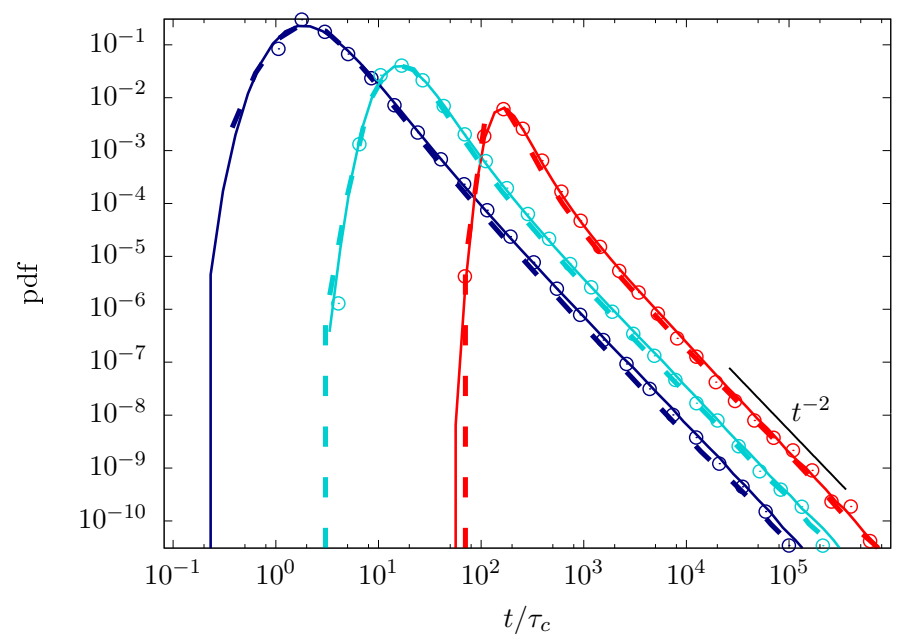

Fig. 3 Breakthrough curves for a flux weighted injection obtained from the DNS (circles), the Bernoulli CTRW (dashed line) and OU CTRW (solid line) at planes located at $6 \ell_{p}, 36 \ell_{p}$, and $200 \ell_{p}$ (from dark blue to red).

\subsubsection{Uniform Injection}

The results are however different when using a uniform injection as initial condition. Under this condition, the particle velocities are non-stationary. Figure 4 displays the breakthrough curves computed at the same distances as in the previous section. The late time slope is very different from the one obtained for the flux-weighted injection. Here, the late time tailing is governed by the initial velocity distribution $p_{0}(v)$, which at small $v$ scales as $p_{0}(v) \propto v^{-0.8}$, see Figure 2 . The initial velocities persist within a distance of about $\ell_{c}$ from the inlet. The long time behavior is dominated by strong particle retention in the vicinity of the inlet and dominated by the transition time over the distance $\ell_{c}$,

$$
\psi_{0}(t)=\frac{\ell_{c}}{t^{2}} p_{0}\left(\ell_{c} / t\right) \propto t^{-1.2}
$$

see also Appendix B.

Both, the Bernoulli and OU models provide good predictions of the breakthrough curves obtained from the DNS. The peak position and width are well captured. Also the behaviors at intermediate and long times are accurately predicted. Both models give the correct long time tailing, while the Bernoulli model slightly overestimates the tail compared to the DNS. This can be traced back to the observation that the Bernoulli model overestimates the persistence of low velocities (Puyguiraud et al., 2019). 


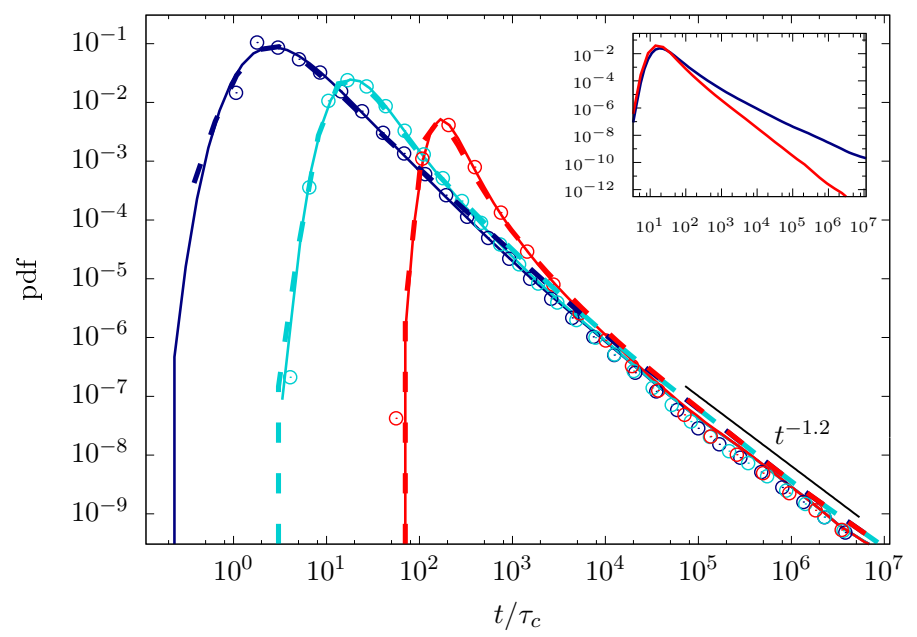

Fig. 4 Breakthrough curves for uniform injection obtained from the DNS (circles), the Bernoulli CTRW (dashed line), and the OU CTRW (solid line) at planes located at $6 \ell_{p}, 36 \ell_{p}$, and $200 \ell_{p}$ (from dark blue to red). Inset: Comparison between the uniform and the flux weighted BTCs for the direct simulation at the plane at $36 \ell_{p}$.

\subsection{Particle Distribution}

In this section we study the evolution of the spatial particle distributions, or propagators $g\left(x_{1}, t\right)$ with focus on the differences in the evolution due to the initial particle distribution. The propagator at a given time maps the spatial heterogeneity of the velocity field which controls the spatial distribution of the mass in the system and, for instance, gives information on the localization of reaction with the solid phase. Thus, together with the breakthrough curve it allows for a spatio-temporal characterization of the solute distribution.

Figure 5 shows $g\left(x_{1}, t\right)$ for uniform and flux-weighted injection conditions at three different times. In both cases, the particle distributions are asymmetric and characterized by a leading edge and long spatial tail. These behaviors are caused by the broad distribution of particle velocities. For the uniform injection, the proportion of particles in low velocity regions is larger than for the flux-weighted injection. Thus, the tailing at short and intermediate times is stronger in the uniform than in the fluxweighted case. With increasing time, the spatial distributions lose the memory of the initial condition and assume the same shape. Note that this is different from the breakthrough curves, whose long time behavior is dominated by the injection condition.

The OU and Bernoulli models predict the spatial profiles under both injection conditions for times $t>\tau_{c}$. For times $t<\tau_{c}$, the stochastic models do not capture the trailing tail in the case of the flux-weighted injection. At times $t<\tau_{c}$, the tail of the spatial distribution in the direct numerical simulation is determined by the velocity components $v_{1}(\mathbf{x})<\ell_{c} / t$ in the mean flow direction, this means by particles that 

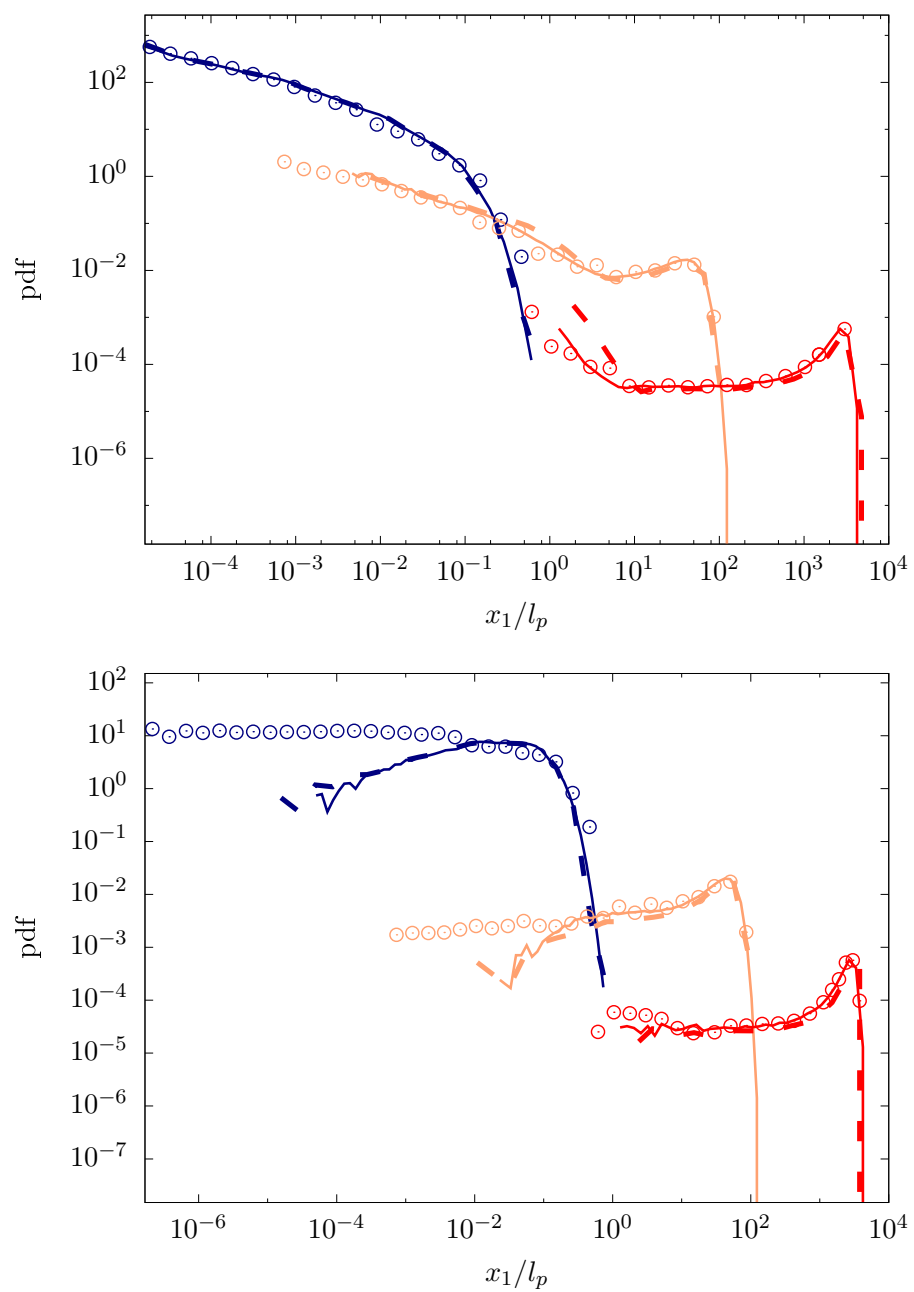

Fig. 5 Evolution of the DNS (circles), OU model (solid lines), and Bernoulli model (dashed lines) mean flow direction propagators for (top panel) uniform injection and (bottom panel) flux-weighted injection at times $t=3.5 \cdot 10^{-1} \tau_{c}$ (blue), $t=3.5 \cdot 10^{2} \tau_{c}$ (orange), and $t=3.5 \cdot 10^{4} \tau_{c}$ (red).

persist in their initial velocity. Thus, if

$$
p_{1}(v)=\int_{\Omega_{0}} d \mathbf{a} \rho(\mathbf{a}) \delta\left[v-v_{1}(\mathbf{a})\right],
$$

is the PDF of the 1-component of the particle velocities in the injection domain, the particle distribution at early times is obtained through the variable transform $x_{1}=v_{1} t$ as

$$
g\left(x_{1}, t\right)=t^{-1} p_{1}\left(x_{1} / t\right) .
$$


In the upscaled stochastic particle model, likewise, the tail of the spatial distribution is due to the particle velocities that persist in their initial velocity. The distance traveled at the initial velocity here, however, is $x_{1}=\chi v_{0} t$ because $v_{0}$ is the initial velocity magnitude. Thus, the upscaled particle model predicts for the early time distribution

$$
g\left(x_{1}, t\right)=\chi t^{-1} p_{0}\left(\chi x_{1} / t\right)
$$

where $p_{0}(v)$ is the PDF of the velocity magnitude in the injection domain. The PDFs of the 1-component and magnitude are in general different, which explains the difference in the tailing behaviors for small times in the case of flux-weighted injection. For the uniform injection, the distributions of the 1-component and the absolute value of velocity are similar in shape, which explains the good match between the stochastic models and the DNS data. While the stochastic models correctly capture the memory of the injection condition on the evolution of the spatial distribution, we do not expect them to be valid at short times and distances, for which the behaviors depend on the local details of the velocity fluctuations.

\subsection{Dispersion}

In this section, we consider the displacement mean and variance. The evolution of the displacement mean is an indicator of t-Lagrangian stationarity, while the displacement variance gives information on particle dispersion. We have seen in the previous section that the Bernoulli and OU models perform equally well in the prediction of the spatial profiles. Thus, here, we compare the DNS data for the displacement mean and variance with the prediction of the stochastic particle model based on the OU model only.

Figure 6 shows the evolution of the mean displacement for uniform and fluxweighted injection conditions. The early time behavior is in both cases linear and given by $\left\langle v_{1}\right\rangle t$, where

$$
\left\langle v_{1}\right\rangle=\int_{-\infty}^{\infty} d v v p_{1}(v)
$$

is the average over the 1-component of the particle velocities in the injection domain. Thus, the slope for the flux-weighted injection is larger than for the uniform injection. At $t>\tau_{c}$, the initial linear behavior crosses over to the long time behavior, which is independent from the injection conditions. For the steady state velocity distribution $p_{s}(v)$ illustrated in Figure 2, for which $p_{s}(v) \approx$ constant at low velocities, CTRW theory predicts $m_{1}(t) \propto t / \ln (t)$ (Comolli and Dentz, 2017). This is confirmed by the DNS data. Both the OU model and the Bernoulli model (not shown) predict the evolution of the mean velocity, with a slight mismatch at short times for the reasons discussed above.

Figure 6 shows the evolution of the displacement variance $\sigma^{2}(t)$ for uniform and flux-weighted initial conditions. At early times, the behaviors are ballistic, this means

$$
\sigma^{2}(t)=\sigma_{v_{1}}^{2} t^{2}
$$



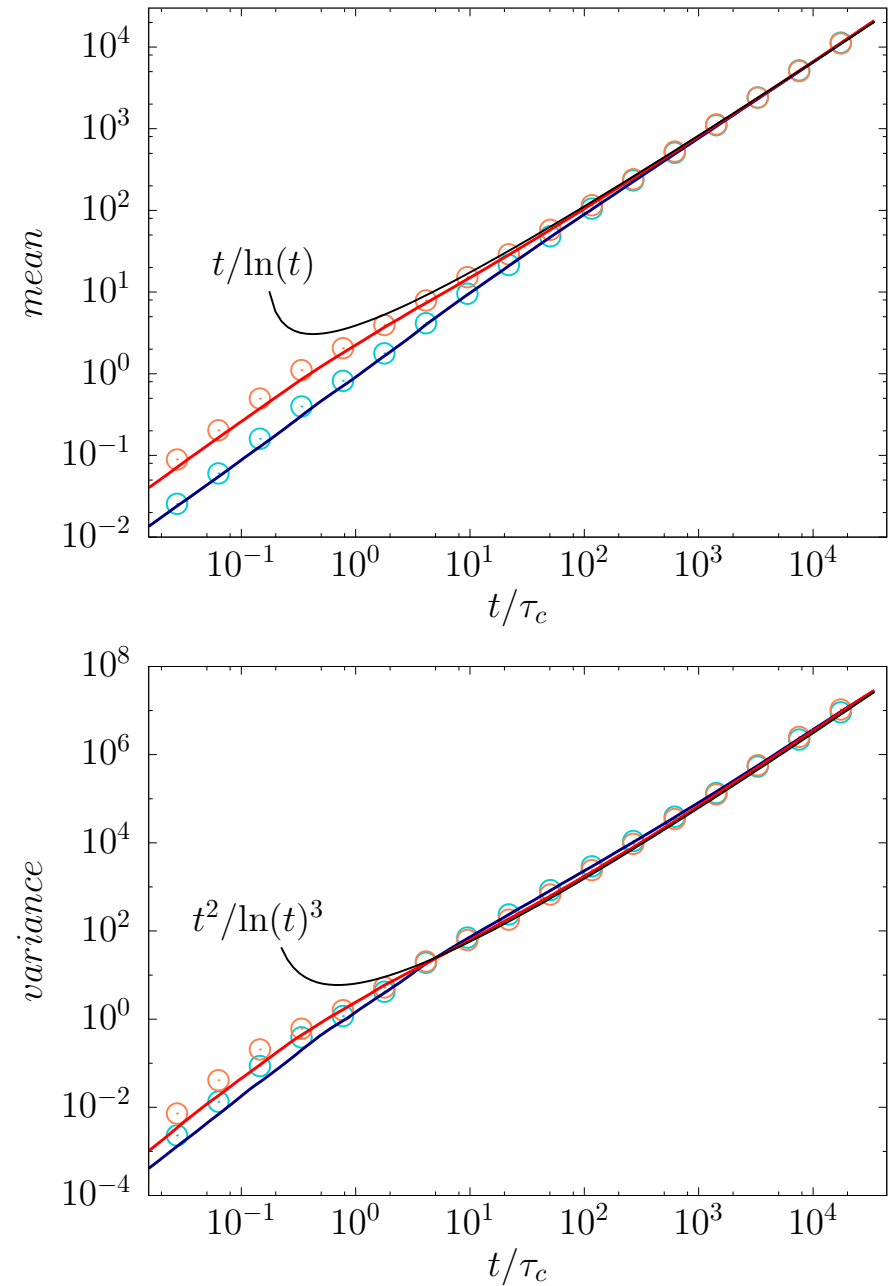

Fig. 6 Evolution of the (top panel) mean displacement and (bottom panel) displacement variance in time for both uniform (blue) and flux-weighted (red) injections. The DNS and OU models results are respectively displayed with open circles and solid lines. The black lines indicate the $t / \ln (t)$ and $t^{2} / \ln (t)^{3} \operatorname{late}$ time scalings.

where $\sigma_{v_{1}}^{2}$ is the variance of $p_{1}(v)$. As, for the mean, the displacement variance is larger for the flux-weighted than for the uniform injection. At $t>\tau_{c}$, the variance crosses over from the ballistic toward the asymptotic regime. For $t \gg \tau_{c}$, CTRW theory predicts $\sigma^{2}(t) \propto t^{2} / \ln (t)^{3}$ (Comolli and Dentz, 2017). The behavior is superdiffusive. Both the OU and Bernoulli (not shown) models predict the evolution of the displacement variance with a mismatch in the ballistic early time behaviors because the stochastic models are determined by the statistics of the velocity magnitude. 


\section{Conclusions}

Using direct three-dimensional pore-scale simulations of flow and transport in a sample of Berea sandstone as a reference case, we have shown that the upscaling of porescale dispersion can be accurately performed using a stochastic approach based on velocity Markov models for equidistantly sampled particle velocities. The upscaled model is implemented in the framework of a time-domain or continuous time random walk approach, which describes particle motion in equidistant spatial steps with random transition times. The presented modeling approach is predictive in the sense that it depends on the Eulerian velocity distribution and advective tortuosity, both flow attributes, and the average pore length which is a medium attribute. It is worth noticing that this dependence allows, in turn, inferring information on the velocity statistics and pore length from (experimental) observations of breakthrough curves, spatial particle distributions and/or displacement moments.

Our analysis has shown that the observed transport behaviors are sensitive to the initial distribution of the tracer particles. Breakthrough curve tailing, for example, can depend on the initial velocity distribution. Under this condition, the breakthrough curve tail gives information on the steady-state velocity distribution in the sample only if the injection domain is already representative (large enough) for the initial velocity distribution to be equal to the stationary PDF, otherwise, the breakthrough curve gives information on the local velocities in the injection domain. Similarly, the spatial particle distribution depends at short and intermediate times on the injection condition. At late times, however, the memory of the initial condition diminishes and the shape becomes independent from the injection condition. This is also reflected in the displacement mean and variance. The early time behaviors of the displacement mean and variance give information on the velocity mean and variance in the injection domain. At late times, the displacement mean shows slightly sublinear behavior, the variance being superlinear, which is due to the tail of the steady state velocity distribution toward low velocity values. In this sense, the evolution of the moments can be seen as a scan through the velocity PDF. At short times, it is dominated by the high and intermediate velocity values, which determine the velocity mean and variance, at long times by the low velocities. The upscaled stochastic particle models can be conditioned on the injection condition through the distribution of initial particle velocities and is able to predict the dependence on the initial condition and full evolution of particle dispersion. We consider two velocity Markov models, the Bernoulli and Ornstein-Uhlenbeck models, which both are parameterized by the velocity correlation length and steady s-Lagrangian velocity PDF. While both models predict the evolution from an initial velocity PDF toward the steady state, they differ in the convergence rates as discussed in (Puyguiraud et al., 2019). Both processes predict the transport behavior and dependence on the initial distribution, which indicates that here the details of the evolution are secondary compared to the fact that there is an evolution.

The presented analysis and the derived stochastic particle models consider purely advective transport. Thus, they are directly relevant for transport scenarios characterized by high Péclet numbers, such as solute transport at high flow rates and passive particles characterized by low diffusion coefficients. The stochastic model is based 
on a Markov model for the streamwise particle velocity, this means that velocities are sampled advectively at a constant frequency in space. The breakthrough curve tailing, for example, is due to the persistence of low velocities over a constant length scale, the pore length. For finite Péclet numbers, particle velocities may be decorrelated due to diffusion across streamlines and low advective transition times may be cut off at the characteristic diffusion time. Thus, we expect anomalous behavior to persist in an intermediate regime depending on the Péclet number and to transition towards normal behavior at times larger than the characteristic pore-scale diffusion time.

Hydrodynamic dispersion and other pore-scale phenomena have their origins in pore-scale velocity fluctuations. Thus, the presented upscaled stochastic model and the associated parameterization can serve as a basis for the systematic quantification of the impact of pore-scale velocity fluctuations on Darcy scale transport phenomena.

\section{A Tortuosity}

We derive here the average of the $\omega_{1}(s, \mathbf{a})$ along a streamline under ergodic conditions. To this end, we first note that the position $x_{1}(s, \mathbf{a})$ can be written by integration of (3) as

$$
x_{1}(s, \mathbf{a})=s\left[\frac{1}{s} \int_{0}^{s} \omega_{1}\left(s^{\prime}, \mathbf{a}\right) d s^{\prime}\right] .
$$

The expression in the square brackets denotes the average of $\omega_{1}(s, \mathbf{a})$ along a particle trajectory. At the same time, it denotes the ratio of linear to streamwise distance,

$$
\left\langle\omega_{1}(s, \mathbf{a})\right\rangle_{s}=\lim _{s \rightarrow \infty} \frac{1}{s} \int_{0}^{s} \omega_{1}\left(s^{\prime}, \mathbf{a}\right) d s^{\prime}=\frac{x_{1}(s, \mathbf{a})}{s},
$$

where the angular brackets with subscript $s$ denote the streamwise average along a trajectory. The average of $\omega_{1}(s, \mathbf{a})$ over an ensemble of particles is defined by

$$
\left\langle\omega_{1}(s, \mathbf{a})\right\rangle=\lim _{V_{0} \rightarrow \infty} \frac{1}{V_{0}} \int_{\Omega_{0}} \frac{v_{1}[\mathbf{x}(s, \mathbf{a})]}{v_{e}[\mathbf{x}(s, \mathbf{a})]} \rho(\mathbf{a}) d \mathbf{a} .
$$

We consider a flux-weighted initial condition, see (4). Under ergodic conditions, this initial condition corresponds to the steady state velocity $\operatorname{PDF} p_{s}(v)$, which is equal to the flux-weighted Eulerian velocity PDF. This can be seen by using

$$
\rho(\mathbf{a})=\frac{1}{V_{0}} \frac{v_{e}(\mathbf{a})}{\left\langle v_{e}(\mathbf{x})\right\rangle} \mathbb{I}\left(\mathbf{a} \in \Omega_{0}\right),
$$

in the limit $V_{0} \rightarrow \infty$. Also, Koponen et al. (1996) pointed out that it is natural for porous media to consider a flux-weighted average, see also Ghanbarian et al. (2013). Furthermore, under ergodic conditions, the average over a single particle trajectory is equal to the average over the initial ensemble of particles and so

$$
\left\langle\omega_{1}(s, \mathbf{a})\right\rangle_{s}=\left\langle\omega_{1}(s, \mathbf{a})\right\rangle=\frac{\left\langle x_{1}(s, \mathbf{a})\right\rangle}{s}=\chi^{-1} .
$$

Using expression (36) in (35), we obtain

$$
\left\langle\omega_{1}(s, \mathbf{a})\right\rangle=\lim _{V_{0} \rightarrow \infty} \frac{1}{V_{0}} \int_{\Omega_{0}} \frac{v_{1}[\mathbf{x}(s, \mathbf{a})]}{v_{e}[\mathbf{x}(s, \mathbf{a})]} \frac{v_{e}(\mathbf{a})}{\left\langle v_{e}(\mathbf{x})\right\rangle} d \mathbf{a} .
$$

In order to evaluate this expression, we perform the variable change $\mathbf{a} \rightarrow \mathbf{x}(s, \mathbf{a})$,

$$
\left\langle\omega_{1}(s, \mathbf{a})\right\rangle=\lim _{V_{0} \rightarrow \infty} \frac{1}{V_{0}} \int_{\Omega(s)} \frac{v_{1}[\mathbf{x}(s, \mathbf{a})]}{v_{e}[\mathbf{x}(s, \mathbf{a})]} \frac{v_{e}(\mathbf{a})}{\left\langle v_{e}(\mathbf{x})\right\rangle} \mathbb{J}(\mathbf{a}, s)^{-1} d \mathbf{x},
$$


where $\mathbb{J}(\mathbf{a}, s)$ is the Jacobian of the transformation. It can be determined by noting that (Batchelor, 2000, p. 75)

$$
\frac{d \mathbb{J}(\mathbf{a}, s)}{d s}=\mathbb{J}(\mathbf{a}, s) \nabla \cdot \frac{\mathbf{v}[\mathbf{x}(s, \mathbf{a})]}{v_{e}[\mathbf{x}(s, \mathbf{a})]}
$$

This differential equation can be integrated by noting that $\nabla \cdot \mathbf{v}(\mathbf{x})=0$ and

$$
\frac{d v_{e}[\mathbf{x}(s, \mathbf{a})]}{d s}=\nabla v_{e}[\mathbf{x}(s, \mathbf{a})] \cdot \mathbf{v}[\mathbf{x}(s, \mathbf{a})]
$$

which follows by using the chain rule and (3). Thus, we obtain for the initial condition $\mathbb{J}(\mathbf{a}, s=0)=1$ that

$$
\mathbb{J}(\mathbf{a}, s)=\frac{v_{e}(\mathbf{a})}{v_{e}[\mathbf{x}(s, \mathbf{a})]} .
$$

Inserting this expression into (38) gives

$$
\left\langle\omega_{1}(s, \mathbf{a})\right\rangle=\lim _{V_{0} \rightarrow \infty} \frac{1}{V_{0}} \int_{\Omega(s)} \frac{v_{1}(\mathbf{x})}{\left\langle v_{e}(\mathbf{a})\right\rangle} d \mathbf{x}=\frac{\left\langle v_{1}\right\rangle}{\left\langle v_{e}\right\rangle} .
$$

This result is consistent with Koponen et al. (1996). This implies that at $s \gg \ell_{p}$, we can set

$$
\left\langle\omega_{1}(s, \mathbf{a})\right\rangle=\chi^{-1}=\frac{\left\langle v_{1}\right\rangle}{\left\langle v_{e}\right\rangle} .
$$

\section{B Continuous Time Random Walk}

For transition length of the order of the correlation length $\ell_{c}$, subsequent particle velocities can be considered independent and thus, the space-time particle motion (13a) may be approximated by

$$
x_{n+1}=x_{n}+\frac{\ell_{c}}{\chi}, \quad t_{n+1}=t_{n}+\tau_{n},
$$

where $x_{n}=x\left(s_{n}\right)$ with $s_{n}=n \ell_{c}$. The random transition time $\tau_{n}$ is given by

$$
\tau_{n}=\frac{\ell_{c}}{v_{s}\left(s_{n}\right)}
$$

The time increments for $n>0$ is distributed as

$$
\psi(t)=\frac{\ell_{c}}{t^{2}} p_{s}\left(\ell_{c} / t\right)
$$

For $n=0$, the transition time PDF is distributed according to

$$
\psi_{0}(t)=\frac{\ell_{c}}{t^{2}} p_{0}\left(\ell_{c} / t\right)
$$

Under steady state conditions, this means for $p_{0}(v)=p_{s}(v)$ and thus $\psi_{0}(v)=\psi(v)$, Equations (45) describe a continuous time random walk as discussed in Berkowitz et al. (2006). Thus, the asymptotic behavior of the breakthrough curves and displacement moments can be predicted based on the scalings of the transition time distribution. For $\psi(t) \propto t^{-1-\beta}$ at large times, the breakthrough curves scales as $f\left(t, x_{1}\right) \propto t^{-1-\beta}$, the mean displacement scales as $m_{1}(t) \propto t$ and the displacement variance as $\sigma^{2}(t) \propto t^{3-\beta}$. Note that this scaling for $\psi(t)$ implies that the velocity distribution $p_{s}(v) \propto v^{\beta-1}$ at small velocities. 


\section{References}

Batchelor, G. K. (2000). An Introduction to Fluid Dynamics. Cambridge University Press.

Bear, J. (1972). Dynamics of fluids in porous media. American Elsevier, New York.

Berkowitz, B., Cortis, A., Dentz, M., and Scher, H. (2006). Modeling non-fickian transport in geological formations as a continuous time random walk. Reviews of Geophysics, 44(2).

Berkowitz, B. and Scher, H. (1995). On characterization of anomalous dispersion in porous and fractured media. Water Resources Research, 31(6), 1461-1466.

Bijeljic, B. and Blunt, M. J. (2006). Pore-scale modeling and continuous time random walk analysis of dispersion in porous media. Water Resources Research, 42(1).

Bijeljic, B., Mostaghimi, P., and Blunt, M. J. (2011). Signature of non-fickian solute transport in complex heterogeneous porous media. Phys. Rev. Lett., 107(20):204502.

Brenner, H. and Edwards, D. (1993). Macrotransport Processes. Butterworth-Heinemann, MA, USA.

Carrel, M., Morales, V. L., Dentz, M., Derlon, N., Morgenroth, E., and Holzner, M. (2018). Pore-scale hydrodynamics in a progressively bioclogged three-dimensional porous medium: 3-d particle tracking experiments and stochastic transport modeling. Water Resources Research, 54(3):2183-2198.

Carrera, J., Sánchez-Vila, X., Benet, I., Medina, A., Galarza, G., and Guimerà, J. (1998). On matrix diffusion: formulations, solution methods and qualitative effects. Hydrogeology Journal, 6(1):178-190.

Cherblanc, F., Ahmadi, A., and Quintard, M. (2007). Two-domain description of solute transport in heterogeneous porous media: Comparison between theoretical predictions and numerical experiments. Advances in Water Resources, 30:1127-1143.

Comolli, A. and Dentz, M. (2017). Anomalous dispersion in correlated porous media: a coupled continuous time random walk approach. The European Physical Journal B, 90(9):166.

Davit, Y., Quintard, M., and Debenest, G. (2010). Equivalence between volume averaging and moments matching techniques for mass transport models in porous media. International Journal of Heat and Mass Transfer, 53(21-22):4985-4993.

De Anna, P., Le Borgne, T., Dentz, M., Tartakovsky, A. M., Bolster, D., and Davy, P. (2013). Flow intermittency, dispersion, and correlated continuous time random walks in porous media. Physical review letters, 110(18):184502.

De Anna, P., Quaife, B., Biros, G., and Juanes, R. (2017). Prediction of velocity distribution from pore structure in simple porous media. Phys. Rev. Fluids, 2:124103.

de Josselin de Jong, G. (1958). Longitudinal and transverse diffusion in granular deposits. Trans. Amer Geophys. Un., 39:67-74.

Dentz, M. and Berkowitz, B. (2003). Transport behavior of a passive solute in continuous time random walks and multirate mass transfer. Water Resources Research, 39(5).

Dentz, M., Borgne, T. L., Englert, A., and Bijeljic, B. (2011). Mixing, spreading and reaction in heterogeneous media: A brief review. Journal of Contaminant Hydrology, 120-121:1 - 17

Dentz, M., Cortis, A., Scher, H., and Berkowitz, B. (2004). Time behavior of solute transport in heterogeneous media: transition from anomalous to normal transport. Adv. Water Resour., 27(2):155-173.

Dentz, M., Icardi, M., and Hidalgo, J. J. (2018). Mechanisms of dispersion in a porous medium. Journal of Fluid Mechanics, 841:851-882.

Dentz, M., Kang, P. K., Comolli, A., Le Borgne, T., and Lester, D. R. (2016). Continuous time random walks for the evolution of lagrangian velocities. Physical Review Fluids, 1(7):074004.

Dentz, M., Kinzelbach, H., Attinger, S., and Kinzelbach, W. (2000). Temporal behavior of a solute cloud in a heterogeneous porous medium: 1. point-like injection. Water Resources Research, 36(12):3591-3604

Devroye, L. (1986). Non-Uniform Random Variate Generation. Springer, New York.

Gardiner, C. (2010). Stochastic Methods. Springer Verlag Berlin-Heidelberg.

Ghanbarian, B., Hunt, A., P. Ewing, R., and Sahimi, M. (2013). Tortuosity in porous media: A critical review. 77:1461.

Gjetvaj, F., Russian, A., Gouze, P., and Dentz, M. (2015). Dual control of flow field heterogeneity and immobile porosity on non-fickian transport in berea sandstone. Water Resources Research, 51(10):82738293.

Haggerty, R. and Gorelick, S. M. (1995). Multiple-rate mass transfer for modeling diffusion and surface reactions in media with pore-scale heterogeneity. Water Resources Research, 31(10):2383-2400

Holzner, M., Morales, V. L., Willmann, M., and Dentz, M. (2015). Intermittent Lagrangian velocities and accelerations in three-dimensional porous medium flow. Phys. Rev. E, 92:013015. 
Jin, C., Langston, P. A., Pavlovskaya, G. E., Hall, M. R., and Rigby, S. P. (2016). Statistics of highly heterogeneous flow fields confined to three-dimensional random porous media. Phys. Rev. E, 93:013122.

Kang, P. K., de Anna, P., Nunes, J. P., Bijeljic, B., Blunt, M. J., and Juanes, R. (2014). Pore-scale intermittent velocity structure underpinning anomalous transport through 3 -d porous media. Geophysical Research Letters, 41(17):6184-6190.

Koponen, A., Kataja, M., and Timonen, J. (1996). Tortuous flow in porous media. Phys. Rev. E, 54:406410.

Le Borgne, T., Bolster, D., Dentz, M., Anna, P., and Tartakovsky, A. (2011). Effective pore-scale dispersion upscaling with a correlated continuous time random walk approach. Water Resources Research, 47(12).

Le Borgne, T. and Gouze, P. (2008). Non-fickian dispersion in porous media: 2. Model validation from measurements at.

Leal, L. G. (2007). Advanced Transport Phenomena: Fluid Mechanics and Convective Transport Processes. Cambridge Series in Chemical Engineering. Cambridge University Press.

Levy, M. and Berkowitz, B. (2003). Measurement and analysis of non-fickian dispersion in heterogeneous porous media. Journal of contaminant hydrology, 64(3):203-226.

Matyka, M., Golembiewski, J., and Koza, Z. (2016). Power-exponential velocity distributions in disordered porous media. Phys. Rev. E, 93:013110.

Meyer, D. W. and Bijeljic, B. (2016). Pore-scale dispersion: Bridging the gap between microscopic pore structure and the emerging macroscopic transport behavior. Physical Review E, 94(1):013107.

Morales, V. L., Dentz, M., Willmann, M., and Holzner, M. (2017). Stochastic dynamics of intermittent pore-scale particle motion in three-dimensional porous media: Experiments and theory. Geophysical Research Letters, 44(18):9361-9371.

Moroni, M., Kleinfelter, N., and Cushman, J. H. (2007). Analysis of dispersion in porous media via matched-index particle tracking velocimetry experiments. Advances in water resources, 30(1):1-15.

Mostaghimi, P., Bijeljic, B., Blunt, M., et al. (2012). Simulation of flow and dispersion on pore-space images. SPE Journal, 17(04):1-131.

Neuman, S. and Tartakovsky, D. (2009). Perspective on theories of non-fickian transport in heterogeneous media. Advances in Water Resources, 32(5):670-680.

Nicolaides, C., Cueto-Felgueroso, L., and Juanes, R. (2010). Anomalous physical transport in complex networks. Phys. Rev. E, 82:055101.

Noetinger, B., Roubinet, D., Russian, A., Le Borgne, T., Delay, F., Dentz, M., De Dreuzy, J.-R., and Gouze, P. (2016). Random walk methods for modeling hydrodynamic transport in porous and fractured media from pore to reservoir scale. Transport in Porous Media, pages 1-41.

Paganin, D., Mayo, S., Gureyev, T. E., Miller, P. R., and Wilkins, S. W. (2002). Simultaneous phase and amplitude extraction from a single defocused image of a homogeneous object. Journal of microscopy, 206(1):33-40.

Painter, S. and Cvetkovic, V. (2005). Upscaling discrete fracture network simulations: An alternative to continuum transport models. Water Resour. Res., 41:W02002.

Pollock, D. W. (1988). Semianalytical computation of path lines for finite-difference models. Ground Water, 26(6):743-750.

Porta, G., Chaynikov, S., Riva, M., and Guadagnini, A. (2013). Upscaling solute transport in porous media from the pore scale to dual-and multicontinuum formulations. Water Resources Research, 49(4):20252039 .

Porta, G. M., Bijeljic, B., Blunt, M., and Guadagnini, A. (2015). Continuum-scale characterization of solute transport based on pore-scale velocity distributions. Geophysical Research Letters, 42(18):75377545.

Puyguiraud, A., Gouze, P., and Dentz, M. (2019). Stochastic dynamics of lagrangian pore-scale velocities in three-dimensional porous media. Water Resources Research, 55, 1196-1217.

Risken, H. (1996). The Fokker-Planck Equation. Springer Heidelberg New York.

Saffman, P. (1959). A theory of dispersion in a porous medium. Journal of Fluid Mechanics, 6(03):321349.

Salles, J., Thovert, J.-F., Delannay, R., Prevors, L., Auriault, J.-L., and Adler, P. (1993). Taylor dispersion in porous media. determination of the dispersion tensor. Physics of Fluids A: Fluid Dynamics, 5(10):23482376

Sanchez, S., Ahlberg, P. E., Trinajstic, K. M., Mirone, A., and Tafforeau, P. (2012). Three-dimensional synchrotron virtual paleohistology: a new insight into the world of fossil bone microstructures. Microscopy and Microanalysis, 18(5):1095-1105. 
Sherman, T., Paster, A., Porta, G., and Bolster, D. (2019). A spatial markov model for upscaling transport of adsorbing-desorbing solutes. Journal of Contaminant Hydrology.

Siena, M., Riva, M., Hyman, J., Winter, C. L., and Guadagnini, A. (2014). Relationship between pore size and velocity probability distributions in stochastically generated porous media. Physical Review E, 89(1):013018.

Smal, P., Gouze, P., and Rodriguez, O. (2018). An automatic segmentation algorithm for retrieving subresolution porosity from x-ray tomography images. Journal of Petroleum Science and Engineering.

Sund, N., Bolster, D., Mattis, S., and Dawson, C. (2015). Pre-asymptotic transport upscaling in inertial and unsteady flows through porous media. Transport in Porous Media, 109(2):411-432.

Sund, N. L., Porta, G. M., and Bolster, D. (2017). Upscaling of dilution and mixing using a trajectory based spatial markov random walk model in a periodic flow domain. Advances in water resources, 103:76-85.

Weller, H. G., Tabor, G., Jasak, H., and Fureby, C. (1998). A tensorial approach to computational continuum mechanics using object-oriented techniques. Computers in physics, 12(6):620-631.

Whitaker, S. (1999). The Method of Volume Averaging. Kluwer Academic Publishers.

Wood, B. D. (2009). The role of scaling laws in upscaling. Advances in Water Resources, 32(5):723 736. Dispersion in Porous Media.

Wright, E., Sund, N., Richter, D., Porta, G., and Bolster, D. (2019). Upscaling mixing in highly heterogeneous porous media via a spatial Markov model. Water, 11(1):53. 